\title{
CUG-BP binds to RNA substrates and recruits PARN deadenylase
}

\author{
KAREN C.M. MORAES, CAROL J. WILUSZ, and JEFFREY WILUSZ \\ Department of Microbiology, Immunology \& Pathology, College of Veterinary Medicine \& Biomedical Sciences, \\ Colorado State University, Fort Collins, Colorado 80523, USA
}

\begin{abstract}
CUG-BP is the human homolog of the Xenopus EDEN-BP, which was shown previously to bind to mRNAs, such as c-mos, that exhibit rapid deadenylation following fertilization of the oocyte. While several studies have focused on roles of CUG-BP as a splicing or translation regulator in mammalian cells, its role in mRNA decay has not been examined in detail. Here, we have used an in vitro deadenylation assay to dissect the function of CUG-BP in the decay of two ARE-containing mRNAs: c-fos and TNF $\alpha$. CUG-BP binds specifically to both of these RNAs and stimulates poly(A) shortening by PARN. Moreover, CUG-BP interacts with PARN in extracts by coimmunoprecipitation, and this interaction can be recapitulated using recombinant proteins. CUG-BP, therefore, is the first RNA-binding protein shown to directly recruit a deadenylase to an RNA substrate.
\end{abstract}

Keywords: deadenylation; mRNA stability; myotonic dystrophy; EDEN; AU-rich

\section{INTRODUCTION}

Deadenylation is a rate-limiting step in the turnover of most eukaryotic mRNAs. In addition, removal of the poly $(\mathrm{A})$ tail is an effective means of inhibiting translation, as the poly $(\mathrm{A})$ binding protein $(\mathrm{PABP})$ is a translation initiation factor (Kahvejian et al. 2005). In Xenopus oocytes, poly(A) tail length is used extensively to control gene expression, as there is virtually no transcription ongoing during oocyte development (Richter 1999). The majority of maternal mRNAs undergo a default deadenylation during the maturation process, and this is executed by the PARN deadenylase (Korner et al. 1998). However, several mRNAs, including c-mos and $\mathrm{x}$-myc II, become polyadenylated during maturation due to the presence of a cytoplasmic polyadenylation element (CPE). Immediately following fertilization, CPE-containing $\mathrm{mRNAs}$ are rapidly deadenylated, leading to translational silencing. In contrast to the default deadenylation pathway, post-fertilization deadenylation is dependent on the presence of cis-acting sequences: AU-rich elements (AREs) and/or EDEN (embryonic deadenylation element) found within the 3'UTR (Richter 1999).

Reprint requests to: Jeffrey Wilusz, Department of Microbiology, Immunology \& Pathology, College of Veterinary Medicine \& Biomedical Sciences, Colorado State University, Fort Collins, CO 80523, USA; e-mail: jeffrey.wilusz@colostate.edu; fax: (970) 491-0603.

Article published online ahead of print. Article and publication date are at http://www.rnajournal.org/cgi/doi/10.1261/rna.59606.
AREs in Xenopus mRNAs appear similar to those in mammalian mRNAs that are able to modulate deadenylation rates. The AREs of either Xenopus x-myc II mRNA or human GM-CSF mRNAs mediate rapid deadenylation following fertilization (Voeltz and Steitz 1998). In mammalian cells, numerous ARE-binding proteins have been identified that either positively or negatively regulate decay and/or translation (Wilusz and Wilusz 2004), and several studies have shown interactions between ARE-binding proteins and the deadenylation machinery (Lai et al. 2003; Gherzi et al. 2004; Lykke-Andersen and Wagner 2005). However, there has been no direct evidence for an interaction between ARE-binding proteins and the deadenylation machinery that leads to modulation of this process.

The EDEN-binding protein, EDEN-BP, is necessary for the function of the EDEN sequence (Paillard et al. 1998). Subsequent studies have determined that EDEN-BP recognizes sequences that are rich in uridine/purine dinucleotides (Bonnet-Corven et al. 2002). Furthermore, EDEN-BP can also bind to AU-rich elements, such as that found in c-jun (Paillard et al. 2002). Interestingly, EDEN-dependent deadenylation appears to be facilitated by the presence of neighboring AU-rich sequences (Audic et al. 1998). As yet, the enzyme responsible for sequence-specific deadenylation following fertilization has not been identified, but it appears to be biochemically distinct from the enzyme that 
carries out the default deadenylation pathway (Paillard et al. 1996). Thus, it is not known how binding of EDEN-BP leads to activation of deadenylation.

EDEN-BP is conserved in higher eukaryotes; Bruno in Drosophila, etr-1 in Caenorhabditis elegans, and CUG-BP in human cells are all homologous to EDEN-BP. CUG-BP is of particular interest, as its overexpression results in a phenotype similar to myotonic dystrophy and is thought to be at least partially responsible for the muscle-specific effects of this disease (Ho et al. 2005). Previous studies of CUG-BP function have focused mainly on the roles of this protein in regulating alternative splicing (Philips et al. 1998) and also on its ability to modulate translation of several mRNAs (Mukhopadhyay et al. 2003; Timchenko et al. 2005). However, as CUG-BP is able to functionally substitute for EDEN-BP to induce deadenylation in Xenopus extracts (Paillard et al. 2003), it seems likely that it also plays a similar role in regulating poly(A) shortening in mammalian cells.

Our lab has previously developed an in vitro assay for assessing the mechanisms and regulation of mRNA decay (Ford et al. 1999). This assay shows accelerated turnover of ARE-containing mRNAs (Ford et al. 1999) as well as cap-dependent deadenylation by PARN (Gao et al. 2000), making it an appropriate system to examine the influence of CUG-BP on deadenylation. In this study, we demonstrate the binding of CUG-BP to two mammalian AREcontaining mRNAs in HeLa cell extracts. CUG-BP appears to recognize both the ARE and EDEN-like sequences in these RNAs. Furthermore, we show that CUG-BP can interact with the PARN deadenylase to promote deadenylation of these substrate RNAs. This is the first evidence for an RNA-binding protein directly recruiting an active deadenylase to its RNA substrate.

\section{RESULTS}

In order to study potential effects of CUG-BP on mRNA deadenylation it was first necessary to identify substrate RNAs that can be recognized by CUG-BP. Recent reports examined the binding specificity of CUG-BP and found that it exhibits a strong preference for UG repeats and UGUU sequences (Takahashi et al. 2000; Faustino and Cooper 2005). CUG-BP has also been shown to associate with CUG repeats (Lu et al. 1999) and AU-rich elements (Mukhopadhyay et al. 2003). We had previously shown that the c-fos and TNF $\alpha$ AU-rich elements can enhance mRNA decay in HeLa extracts (Ford et al. 1999), so these seemed appropriate candidates. We examined the regions flanking the AREs of these two RNAs for other sequences that might be recognized by CUG-BP based on the findings of the studies mentioned above. Those we found are highlighted in Figure 1. In addition to the ARE, both c-fos and TNF $\alpha$ RNAs contain multiple UGUA, UGUU, and UGUG sequences that could perhaps be recognized by CUG-BP. The occurrence of these sequences is significantly higher (nine in c-fos and seven in TNF $\alpha$ ) than might be predicted at random in a $250 \mathrm{nt}$ RNA (2.9 occurrences expected). The presence of potential CUG-BP binding sites and AU-rich elements in the same RNA is significant, as AU-rich sequences were shown to enhance the function of the EDEN element in the Xenopus system (Audic et al. 1998). Moreover, the mammalian c-jun ARE was shown to induce

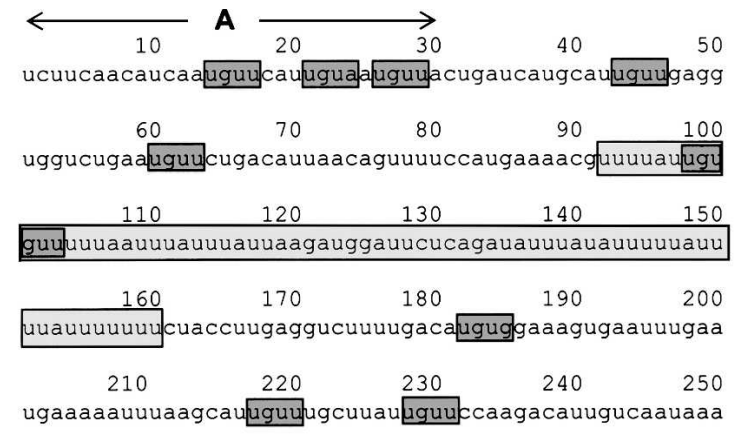

c-fos

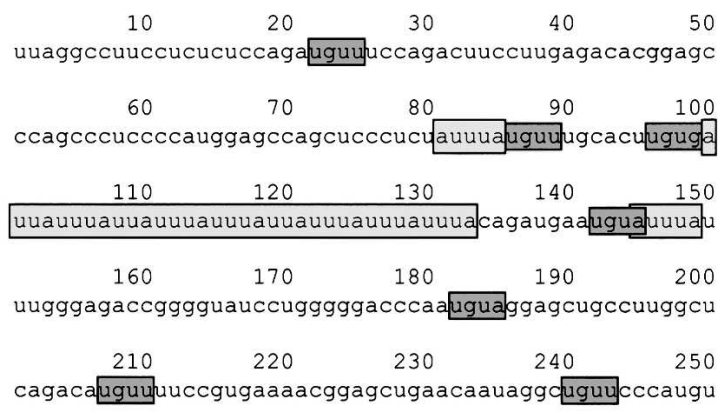

TNF $\alpha$

\section{AU-rich element}

putative CUG-BP site

aquguvuaua uquauguguugue una ugugugugugugugcucuucu EDEN RNA

FIGURE 1. Mammalian ARE-containing mRNAs contain candidate binding sites for CUG-BP. The sequences of c-fos and TNF $\alpha$ RNA substrates are depicted, with AU-rich elements and putative CUG-BP binding sites highlighted. In addition, the region of the c-fos RNA with the best matches to CUG-BP consensus is labeled (A). The sequence of the EDEN region of c-mos, which is used as a competitor in Figure 3, is also shown. 
EDEN-dependent deadenylation in Xenopus embryos (Paillard et al. 2002).

\section{CUG-BP binds to sequences from TNFa and c-fos mRNAs}

It was important to determine whether CUG-BP actually bound to the candidate substrate RNA in extracts. Radiolabeled $\mathrm{TNF} \alpha$, c-fos RNAs, and a control RNA(Gem) derived from the pGem 4 polylinker were generated by in vitro transcription and incubated in HeLa cytoplasmic extracts. The Gem control RNA was chosen in part because it lacks any sequences with homology with known CUG-BP binding sites. UV-cross-linking was used to visualize proteins bound to each RNA, and the presence of CUGBP was determined by immunoprecipitation of the crosslinked bands. As seen in Figure 2A, CUG-BP bound to both the TNF $\alpha$ RNA and the c-fos RNA substrates, as predicted, but not to the control RNA. Immunodepletion using CUG$\mathrm{BP}$-specific antiserum significantly reduced the level of CUG$\mathrm{BP}$ in the extracts (Fig. 2A, ID lanes). Therefore, CUG-BP specifically binds the TNF $\alpha$ and c-fos RNA substrates.
We next examined the specificity of CUG-BP binding to c-fos RNA in more detail using unlabeled RNA competitors. As shown in Figure 2B, CUG-BP was efficiently competed by addition of $50 \mathrm{fmol}$ or more of unlabeled EDEN RNA oligo, but not by a matched oligo in which the EDEN binding sites had been mutated. This is consistent with previous data showing that CUG-BP exhibits similar binding specificity to EDEN-BP. In addition, we were able to compete CUG-BP from the c-fos RNA using an RNA oligo containing just the 34-nt AU-rich sequence from TNF $\alpha$ (Fig. 2C). However, the TNF $\alpha$ ARE oligo was a weaker competitor than the EDEN oligo requiring between 6.7 and 34 pmol to completely compete the CUG-BP band. This suggests that CUG-BP has a higher affinity for uridine/ purine-rich sequences than for AU-rich sequences.

As both TNF $\alpha$ and c-fos RNA substrates contain AU-rich sequences as well as other potential CUG-BP binding sites, we wished to test whether CUG-BP bound to the ARE or elsewhere in the RNA. We deleted the ARE region from each RNA and repeated the UV cross-linking experiment. As seen in Figure 3A, deletion of the ARE had no discernable effect on CUG-BP cross-linking in either case. This
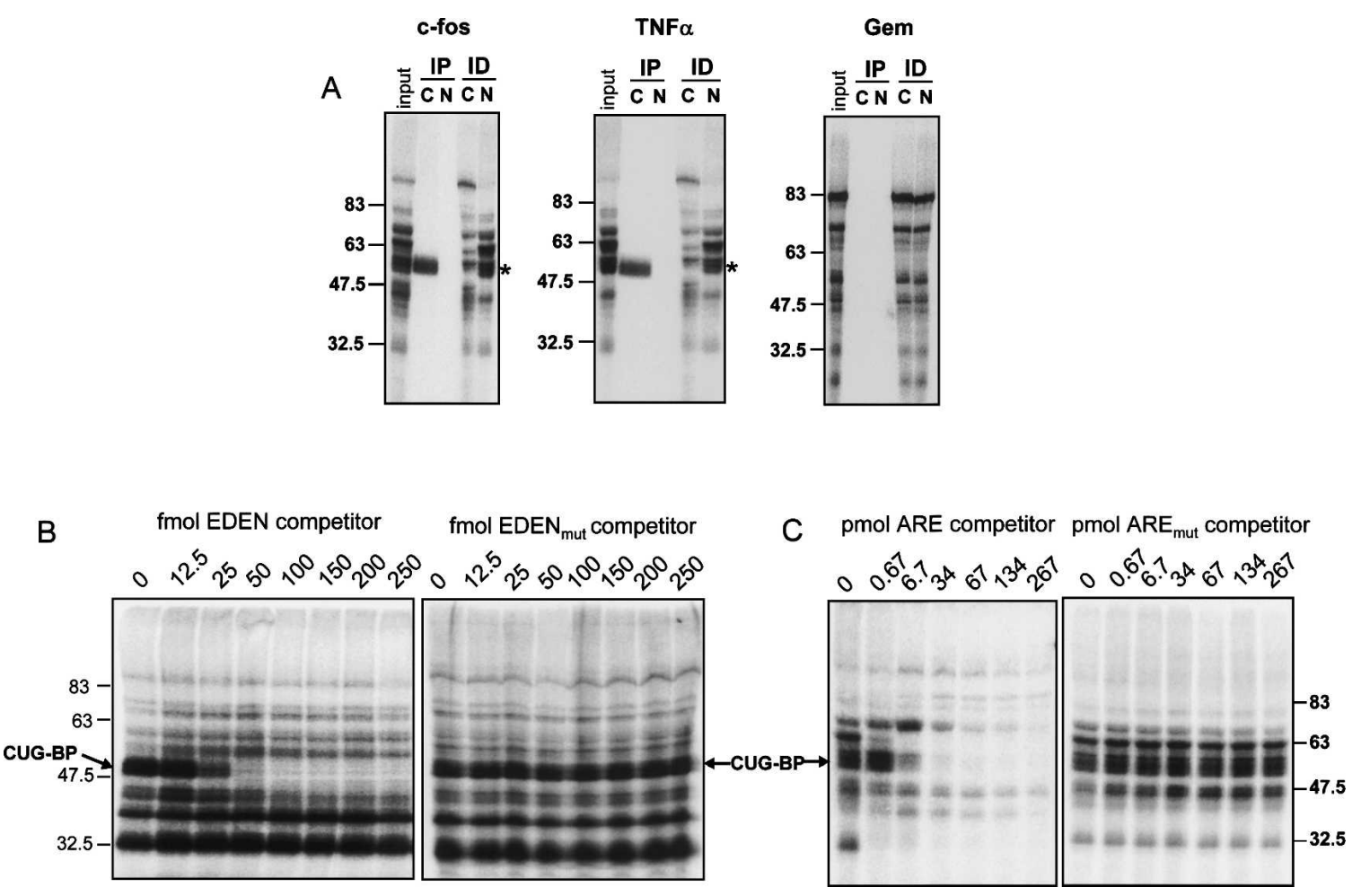

FIGURE 2. The c-fos and TNF $\alpha$ RNAs cross-link specifically to CUG-BP in vitro. (A) The indicated radiolabeled substrate RNA was incubated in extracts and irradiated with UV light to cross-link proteins to the RNA (input). A portion of the cross-linked proteins were subjected to immunoprecipitation $(I P)$ with anti-CUG-BP antibody $(C)$, or normal serum $(N)$. In a separate experiment $(I D)$, anti-CUG-BP antibody $(C)$, or normal serum $(N)$, were used to immunodeplete the extracts following cross-linking and the supernatants were analyzed. The products were separated by SDS-PAGE. The position of the cross-linked CUG-BP is indicated. (B) UV cross-linking to the c-fos RNA substrate was performed in the presence of increasing femtomolar amounts of EDEN RNA competitor or a control RNA $\left(\right.$ EDEN $\left._{\text {mut }}\right)$, in which the CUG-BP binding sites had been mutated. The CUG-BP band is indicated. (C) UV cross-linking to the c-fos RNA substrate was performed in the presence of increasing picomolar amounts of TNF $\alpha$ ARE competitor or a control RNA in which the AUUUA pentamers had been mutated. The CUG-BP band is indicated, and molecular weight markers are shown. 


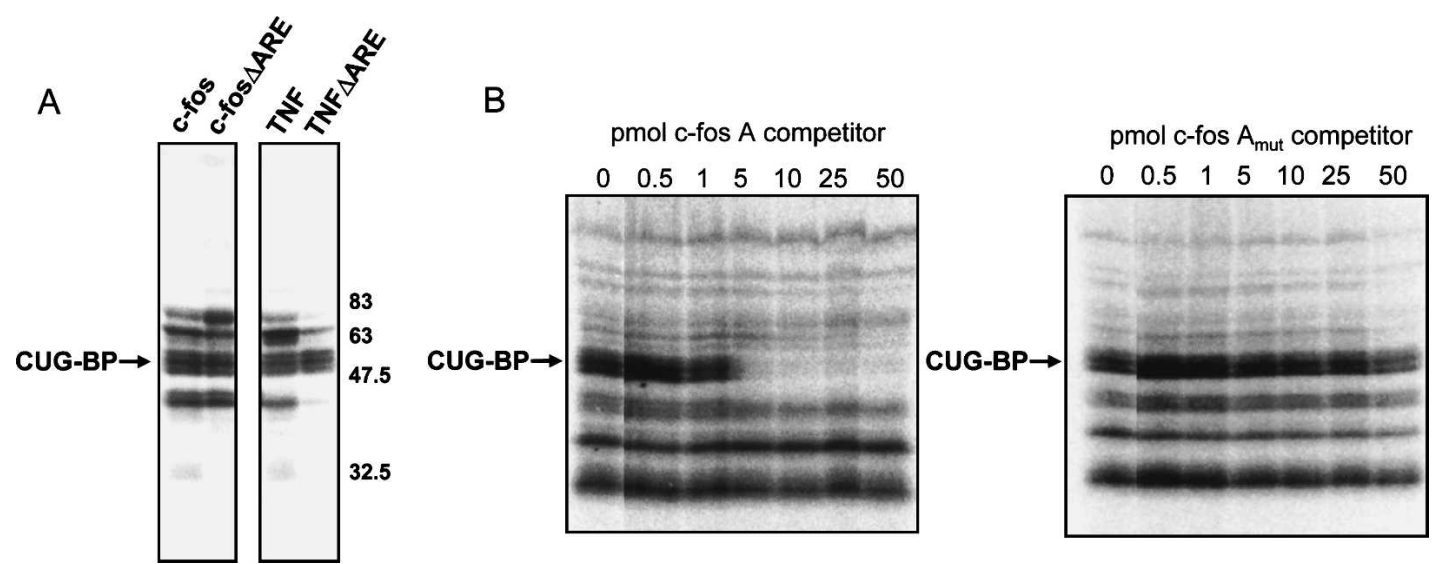

FIGURE 3. CUG-BP binds to AREs and to sites outside those regions. (A) UV cross-linking was performed using c-fos and TNF $\alpha$ RNA substrates and variant RNAs that lacked the ARE. The CUG-BP band and molecular weight markers are indicated. $(B)$ Increasing picomolar amounts of Region A of the c-fos RNA (see Fig. 1) or a mutant version were used as competitor RNAs in UV cross-linking reactions. The position of the CUG-BP band is indicated.

strongly suggests that CUG-BP is binding to sequences other than the ARE, although it remains possible that the AREs are recognized as well. The best candidate EDEN-like sequence in c-fos lies at the $5^{\prime}$ end of the substrate RNA (labeled " $\mathrm{A}$ " in Fig. 1). This region has two UGUU sequences and a UGUA sequence in a 16-nt stretch. We therefore used an RNA oligo spanning this region as a cold competitor. As predicted, c-fos region A was able to compete CUG-BP effectively from the c-fos RNA substrate, but a mutant version in which the two UGUU sequences were changed to AGAA had no effect (Fig. 3B). Although c-fos region $\mathrm{A}$ is not as efficient a competitor as the EDEN RNA, this is not unexpected, as the EDEN RNA oligo has more and better matches to the optimal CUG-BP binding site. This result shows that CUG-BP is able to bind c-fos region A, probably by recognizing the UGUU sequences, but does not preclude that it may also bind to other regions of this RNA, including perhaps the ARE.

\section{CUG-BP is required for rapid deadenylation}

Having ascertained that CUG-BP could bind to the TNF $\alpha$ and c-fos substrate RNAs, we next wished to test whether CUG-BP binding influences the deadenylation of these RNAs. We again used the artificial Gem RNA as a control, as it did not cross-link to CUG-BP, but it still undergoes rapid deadenylation in our assay with a pattern similar to that of the TNF $\alpha$ RNA. Each RNA was transcribed with a 60-base poly(A) tail and incubated in our in vitro assay. As seen in Figure 4, during the time course of the reaction the poly(A) tail is shortened and a deadenylated intermediate accumulates. It is interesting to note that the TNF $\alpha$ and c-fos RNAs deadenylate with different patterns. TNF $\alpha$ exhibits processive deadenylation with completely deadenylated intermediates accumulating while fully adenylated RNA is still present (Fig. 4A). In contrast, the c-fos RNA substrate undergoes a more distributive poly(A) shortening, with the whole population undergoing deadenylation simultaneously. This reflects the patterns of deadenylation seen for these two mRNAs in vivo (Xu et al. 1997).

To determine the role of CUG-BP in the deadenylation of these RNAs, we added excess EDEN RNA oligo to compete off CUG-BP and performed the same deadenylation assay. This resulted in significant retardation of the deadenylation process for both the TNF $\alpha$ and c-fos RNA substrates such that both RNAs deadenylated in a much

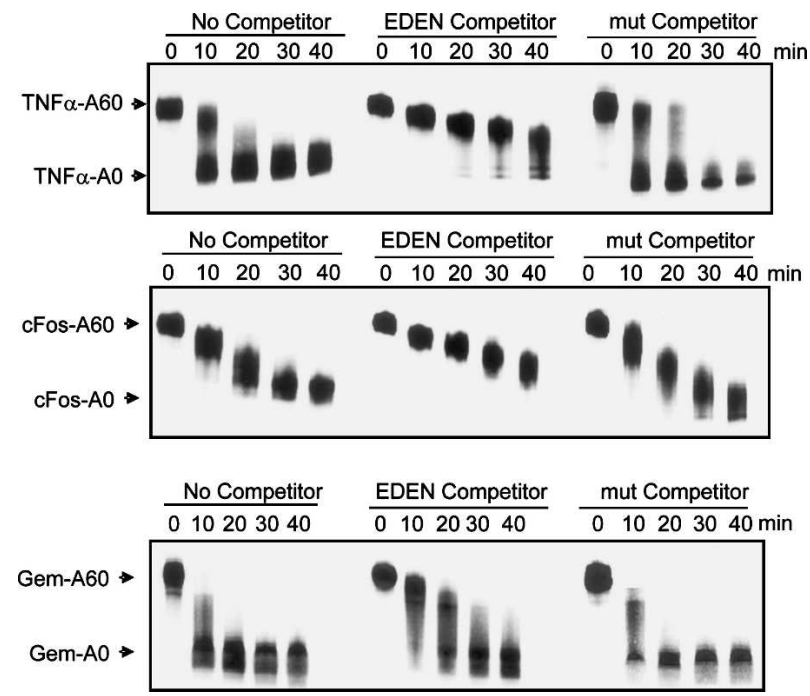

FIGURE 4. Addition of excess EDEN RNA results in inhibition of deadenylation. Deadenylation assays were performed for the times indicated in the presence of no competitor (left), $250 \mathrm{fmol}$ of EDEN RNA competitor (center), or $250 \mathrm{fmol}$ of mutant EDEN competitor (right). Deadenylation of TNF $\alpha$ (upper panel), c-fos (middle panel), and Gem (lower panel) RNAs was visualized following separation on a $4 \%$ denaturing polyacrylamide gel. The migration positions of fully adenylated input RNA and deadenylated products are indicated on the left. 
slower, distributive fashion. In contrast, the EDEN oligo had only a minimal effect on the deadenylation of the Gem control RNA (Fig. 4, lower panel). A mutant EDEN oligo had no effect on the deadenylation rate of any of the three RNA substrates. From this we can conclude that a protein binding to the EDEN sequence, probably CUG-BP, is required for deadenylation in these extracts. The slight effect of the EDEN oligo on the Gem control RNA may be due to sequestration of the PARN deadenylase by the oligo (see below; Figs. 5A, 6).

In order to confirm that the effect of the EDEN oligo was due to sequestration of CUG-BP, we used antibodies to CUG-BP to deplete $\sim 70 \%$ of the protein from the extracts prior to performing the deadenylation assay (Fig. 5A). Immunodepletion of CUG-BP had an identical effect to addition of the EDEN oligo-deadenylation of both c-fos and TNF $\alpha$ RNAs was drastically slowed (Fig. 5B), but deadenylation of the Gem control RNA was unaffected (Fig. 5C). Mock depletion with normal serum had no effect. Importantly, we were able to reactivate deadenylation of the TNF $\alpha$ and c-fos RNAs by adding recombinant CUG-BP to the immunodepleted extracts (Fig. 5B). Taken together, these results suggest that CUG-BP is required to recruit the deadenylase to these RNA substrates.

\section{CUG-BP interacts with the PARN deadenylase}

The data described above show that CUG-BP enhances deadenylation of the RNA substrates that it binds to. One way that CUG-BP could achieve this is by directly interacting with the deadenylase and recruiting it to the RNA. In HeLa cytoplasmic extracts the major detectable deadenylase activity is PARN (Gao et al. 2000). We therefore surmised that CUG-BP and PARN may form a complex. This hypothesis is supported by the observation that PARN is codepleted from the extracts during CUG-BP immunodepletion (Fig. 5A, middle panel). We confirmed this interaction in the extracts by immunoprecipitating CUG-BP and looking for PARN in the immunoprecipitate by Western blotting. As seen in Figure 6A, PARN is clearly detectable in the anti-CUG-BP immunoprecipitate but not in that from normal serum. Therefore, PARN and CUG-BP do indeed form a complex in extracts. Moreover, this interaction must be mediated by proteins and not through
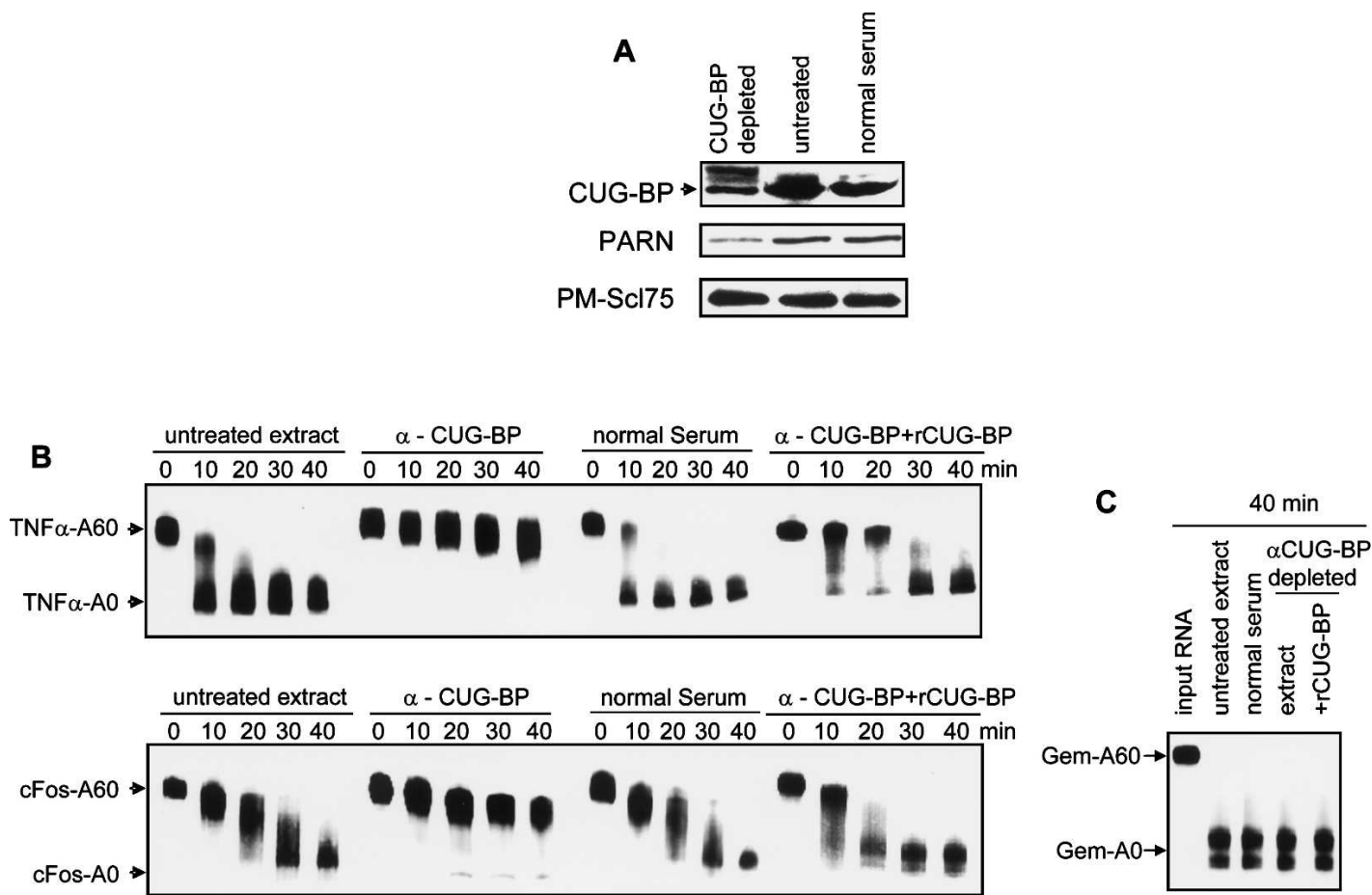

FIGURE 5. CUG-BP is required for deadenylation of TNF $\alpha$ and $c$-fos RNAs. (A) Extracts were depleted using anti-CUG-BP antibodies or normal serum, and CUG-BP levels were determined by Western blotting. The extent of depletion was confirmed by comparison with untreated extract (untreated lane). The band above the CUG-BP band is from immunoglobulin. The same extracts were assayed for levels of PARN before and after CUG-BP depletion by Western blotting using anti-PARN antibodies (middle panel). Blots were reprobed with anti-PMScl75 antibodies as a loading control (lower panel). ( $B$ ) Extracts were either untreated, depleted for CUG-BP ( $\alpha$-CUG-BP), or mock-depleted (normal serum) and used in deadenylation assays over the time course indicated. Where indicated, $30 \mathrm{ng}$ of recombinant CUG-BP $(\alpha-C U G-B P+$ rCUG-BP) was added back to the extract prior to the assay. Deadenylation for TNF $\alpha$ (upper panel) and c-fos (lower panel) RNAs was assessed over the time course indicated. The migration positions of the fully adenylated and deadenylated RNAs are shown. (C) Extracts were treated as in $B$ and deadenylation of the Gem-A60 control RNA was assessed following incubation in the extracts for 40 min. The migration positions of the fully adenylated and deadenylated RNAs are shown. 
A
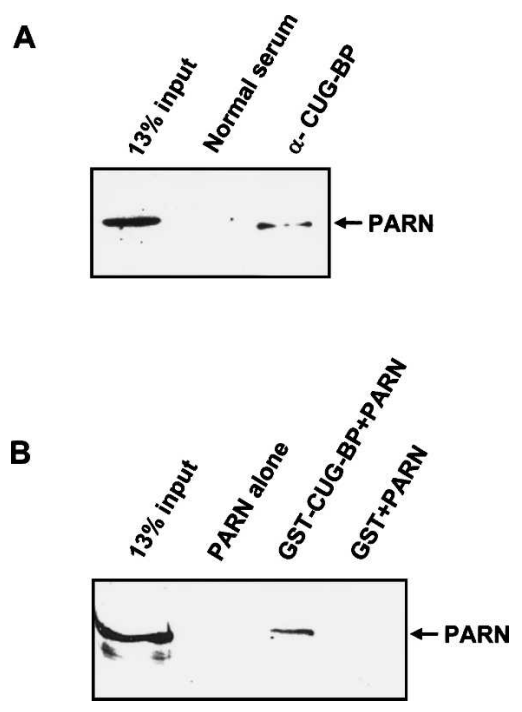

FIGURE 6. (A) CUG-BP and PARN coimmunoprecipitate. CUG-BP was immunoprecipitated from HeLa extracts with anti-CUG-BP antibodies and the immunoprecipitate was separated on a $10 \%$ gel containing SDS and tested for the presence of PARN by Western blotting. A mock immunoprecipitation was performed with normal serum as a control. (B) CUG-BP and PARN interact directly. Recombinant 6HisPARN was incubated in the presence of RNAse A with glutathione Sepharose beads (PARN alone) or with similar beads that had been prebound with GST-CUG-BP (GST-CUG-BP + PARN) or GST (GST + PARN). After extensive washing, the bound proteins were separated by SDS-PAGE and PARN was detected by Western blot.

interaction of both factors with the same RNA substrate as the extracts were treated with RNAse A prior to the immunoprecipitation.

The interaction between CUG-BP and PARN could be direct or mediated by other proteins. In order to distinguish these possibilities, we used recombinant GST-CUG$\mathrm{BP}$ and $6 \mathrm{His}-\mathrm{PARN}$ in a GST pull-down assay. 6His-PARN was incubated with glutathione Sepharose beads or with similar beads that had been prebound to GST or GST-CUGBP. The PARN protein bound to the beads was then detected by Western blotting. As seen in Figure 6B, PARN bound only to the GST-CUG-BP and exhibited no nonspecific affinity for GST or the glutathione beads. As this experiment was performed in the presence of RNAse A, this result shows that the PARN and CUG-BP proteins interact directly.

\section{DISCUSSION}

Our results show that PARN can be recruited to an RNA substrate directly through interaction with CUG-BP bound to that RNA. Although other proteins have been suggested to recruit deadenylases to ARE-containing RNAs, namely tristetraprolin (TTP) (Lai et al. 2003; Lykke-Andersen and Wagner 2005) and KSRP (Gherzi et al. 2004), this is the first time that an interaction between an RNA binding protein and a deadenylase has been shown to affect deadenylation rates.
CUG-BP appears to bind to our two RNA substrates, c-fos and TNF $\alpha$, through EDEN-like sequences that are scattered throughout the RNAs. However, binding of CUG$\mathrm{BP}$ can be also be inhibited by addition of excess cold ARE-containing RNA, indicating that CUG-BP may also recognize the AREs in our substrate RNAs. It is possible that multiple CUG-BP monomers are binding cooperatively to several binding sites in each of these substrates to facilitate efficient recruitment of the deadenylase. In support of this hypothesis, EDEN-BP, the Xenopus homolog of CUG-BP, is reported to dimerize (Bonnet-Corven et al. 2002).

The effect of CUG-BP appears to be to increase the processivity of PARN as depletion or competition of CUG$\mathrm{BP}$ results in a much slower, more synchronous deadenylation pattern (Fig. 4). This is particularly striking in the case of the TNF $\alpha$ RNA substrate, which usually undergoes highly asynchronous deadenylation with unadenylated RNA accumulating in the first time point while some fully adenylated RNA still remains. In contrast, upon depletion of CUG-BP, the deadenylation pattern changes drastically with the entire population of RNA now undergoing slow, synchronous poly $(\mathrm{A})$ shortening. This change in pattern is not due to codepletion of PARN, as addition of recombinant PARN to the depleted extract has very little effect on the deadenylation pattern (data not shown). CUG-BP must be restored to the extract in order to induce rapid asynchronous poly(A) removal (Fig. 5B). Our results suggest that CUG-BP acts to anchor PARN to the RNA substrate, thus reducing the off-rate of the protein and increasing its processivity.

It is useful to draw some comparisons between the regulation of PARN by CUG-BP and the effects of EDENBP on deadenylation in Xenopus embryos. As mentioned above, during oocyte maturation, PARN is responsible for the default deadenylation of mRNAs. Following fertilization, several polyadenylated RNAs including c-mos and Eg2 undergo rapid deadenylation that requires EDEN-BP. Intriguingly, the default pathway, seen prior to fertilization, induces slow, synchronous poly(A) shortening (Legagneux et al. 1995), similar to that seen in our extracts following depletion of CUG-BP. In contrast, EDEN-dependent deadenylation is more rapid and asynchronous (Legagneux et al. 1995), similar to that of TNF $\alpha$ in our untreated extracts. Although the deadenylase responsible for EDEN-dependent deadenylation has not yet been identified, our data suggest that it may well be PARN. There appear to be significant changes in phosphorylation state of EDEN-BP during oocyte maturation and following fertilization (Detivaud et al. 2003). These may well correlate with its ability to interact with PARN and thereby influence deadenylation. There are already indications that CUG-BP phosphorylation state influences its subcellular localization (Roberts et al. 1997). In the future, it will be interesting to determine whether phosphorylation of CUG-BP affects its ability to interact with PARN. 
CUG-BP was first identified as a protein able to bind to the CUG repeats found in the $3^{\prime}$ UTR of the DMPK gene implicated in type I myotonic dystrophy (Timchenko et al. 1996). Overexpression of CUG-BP has since been shown to mimic several of the phenotypes of this debilitating disease in mice, including effects on muscle-specific splicing (Ho et al. 2005). Our results suggest that another mechanism should also be considered; reduced stability of specific mRNAs due to enhanced deadenylation. The implication of our data is that overexpression of CUG-BP could result in binding to inappropriate target mRNAs inducing their decay. Future experiments will test this hypothesis by assessing decay rates of mRNAs in cells overexpressing CUG-BP.

\section{MATERIALS AND METHODS}

\section{Plasmid and DNA template constructions}

The 250-nt fragments of c-fos and TNF $\alpha$ 3'UTRs were generated by RT-PCR using standard conditions and cloned between the EcoRI and PstI sites of pGem-4 (Promega). These plasmids were used as templates to generate the $\triangle \mathrm{ARE}$ mutants, which lacked the sequences indicated as ARE in Figure 1. A ligation-PCR approach was utilized to generate templates for RNAs bearing a 60-nt poly(A) tail (Ford et al. 1999).

\section{Substrate RNAs and competitor RNAs}

RNAs were transcribed in vitro using SP6 RNA polymerase and purified on denaturing acrylamide gels. Transcripts were capped cotranscriptionally by the addition of $5 \mathrm{mM}{ }^{7 \mathrm{me}} \mathrm{GpppG}$ to the reaction.

Synthetic RNAs used in competition analyses were prepared by Dharmacon or IDT. The specific ARE competitor contained a 34-nt AU-rich sequence derived from TNF $\alpha$ 3'UTR (Fig. 1). The corresponding $\mathrm{ARE}_{\text {mut }}$ had the sequence 5'-GGAUUAAC UAAUUGAUACCGCGUAUACACGCGG-3'. The sequence of the EDEN competitor RNA derived from c-mos is shown in Figure 1. The sequence of the EDEN $\mathrm{mut}_{\text {mut }}$ oligo was 5'- AGUCUUUUAUAU CUAUCUCUUCUUUUAUCUCUCUCUCUCUCCUCUUCU-3'. The sequence corresponding to $\mathrm{c}$-fos region A is shown in Figure 1, and the mutant RNA had the UGUU sequences changed to AGAA.

\section{Preparation of cell extracts and in vitro assay}

HeLa S100 cytoplasmic extracts were prepared as described previously (Dignam et al. 1983; Ford and Wilusz 1999). Fifty (50) fmol of polyadenylated RNAs radiolabeled internally at U-residues were incubated in the extracts $(\sim 40 \mu \mathrm{g})$ at $30^{\circ} \mathrm{C}$ in the presence of exogenous poly(A) as described previously (Ford et al. 1999). RNA competitors were added where indicated, and the reaction products were analyzed on $4 \%$ polyacrylamide gels containing $7 \mathrm{M}$ urea.

\section{UV cross-linking/immunoprecipitation/competition assays}

UV cross-linking analyses were performed as described using 50 fmol substrate RNA (Wilusz and Shenk 1988). Cross-linking assays were done in the presence of $25 \mathrm{mM}$ EDTA to inhibit-RNA turnover and allow for accurate comparisons between samples. After digestion with RNase A, cross-linked proteins were analyzed on $10 \%$ SDS acrylamide gels.

For immunoprecipitation analysis following UV cross-linking, $400 \mu \mathrm{L}$ of Net2 buffer $(50 \mathrm{mM}$ Tris at $\mathrm{pH} 7.6,75 \mathrm{mM} \mathrm{NaCl}$, $0.05 \% \mathrm{NP}-40)$ was added to samples, and they were incubated at $4{ }^{\circ} \mathrm{C}$ with anti-CUG-BP antibodies for $3 \mathrm{~h}$. Antigen-antibody complexes were collected on protein A-positive Staphylococcus aureus cells and washed five times with Net 2 buffer. The immunoprecipitated cross-linked proteins were separated on a $10 \%$ SDS acrylamide gel.

The UV cross-linking/competition analyses were done as described above using the indicated amounts of specific or nonspecific cold competitor RNAs.

\section{Immunodepletion and coimmunoprecipitation}

HeLa cytoplasmic extracts were immunodepleted with either control rabbit serum or an anti-CUG-BP monoclonal antibody (Upstate Biotechnology). Briefly, immunodepletions were performed using $3 \mu \mathrm{L}$ of antisera and $20 \mathrm{U}$ of RNAsin. Immune complexes were precipitated using Protein A Sepharose, and depleted extracts were used in the in vitro mRNA decay system described above. In order to check the efficiency of the immunodepletion, a fraction of the immunodepleted extract was loaded on a $10 \%$ SDS polyacrylamide gel and subjected to Western blotting using, in order, rabbit anti-PARN antiserum (1:2000; supplied by M. Wormington), anti-CUG-BP (1:5000; Upstate Biotechnology), and anti-PMSCL 75 (1:10,000; Mukherjee et al. 2002).

For the coimmunoprecipitation of PARN with CUG-BP, $60 \mu \mathrm{L}$ of HeLa cytoplasmic extract was first precleared by the incubation with $20 \mu \mathrm{L}$ of a $50 \%$ slurry of Protein A Sepharose for $30 \mathrm{~min}$ at $4^{\circ} \mathrm{C}$. The precleared extract was then incubated with $\alpha$-CUG-BP $\alpha$-Normal serum in $1 \mathrm{~mL}$ of buffer $\mathrm{B}(300 \mathrm{mM}$ HEPES at $\mathrm{pH}$ 7.9/1.5 mM MgCl$/ 10 \mathrm{mM} \mathrm{KCl}$ ) containing 0.5 mM DTT for $2 \mathrm{~h}$ at $4^{\circ} \mathrm{C}$. The incubation was performed in the presence of $20 \mu \mathrm{g}$ of RNase A. After this first incubation, $18 \mu \mathrm{L}$ of a $50 \%$ slurry of Protein A Sepharose was added; the mixture was incubated for another hour at $4^{\circ} \mathrm{C}$. The beads were then washed three times with NET 2 buffer ( $50 \mathrm{mM}$ Tris at $\mathrm{pH} 7.6,75 \mathrm{mM} \mathrm{NaCl}, 0.05 \%$ Nonidet $\mathrm{P}-40$ ) for $5 \mathrm{~min}$ at $4^{\circ} \mathrm{C}$, and bound proteins were eluted by addition of $2 \times$ SDS gel loading buffer and boiling for $5 \mathrm{~min}$. Following centrifugation, the supernatant was collected and proteins separated on a $10 \%$ SDS polyacrylamide gel. PARN was detected by Western blotting using rabbit anti-PARN antiserum as described above.

\section{Recombinant proteins and GST pull-down assay}

CUG-BP was amplified by RT-PCR and cloned into pGEX-2T. GST-tagged CUG-BP was produced in BL21(DE3) cells and purified on glutathione Sepharose 4B. 6His-tagged PARN was expressed and purified as described previously (Gao et al. 2000).

For the GST pull-down assay, excess GST or GST-CUG-BP fusion protein were bound to $10 \mu \mathrm{L}$ of a $50 \%$ slurry of glutathione Sepharose $4 \mathrm{~B}$ beads in $600 \mu \mathrm{L}$ of PBS for $2 \mathrm{~h}$ at $4^{\circ} \mathrm{C}$. After removal of unbound GST proteins by washing with PBS, the beads were incubated with $200 \mathrm{ng}$ of recombinant $6 \mathrm{His}-\mathrm{PARN}$ in $600 \mu \mathrm{L}$ of PBS buffer containing $0.2 \%$ Triton $\mathrm{X}-100$ at $4^{\circ} \mathrm{C}$ for $1 \mathrm{~h}$. This 
incubation was performed in the presence of $10 \mu \mathrm{g}$ of RNAse A. The beads were then washed three times with PBS containing $0.2 \%$ Triton X-100 and eluted by addition of $2 \times$ SDS gel loading buffer and boiling for $5 \mathrm{~min}$. Following centrifugation, the supernatant was collected and proteins separated on a $10 \%$ SDS polyacrylamide gel. PARN was detected by Western blotting using rabbit anti-PARN antiserum (a kind gift from M. Wormington).

\section{ACKNOWLEDGMENTS}

This research was supported by an award to J.W. from the $\mathrm{NIH}$ (GM063832). We thank Maurice Swanson and Michael Wormington for supplying antibodies to CUG-BP and PARN, respectively.

Received February 10, 2006; accepted February 28, 2006.

\section{REFERENCES}

Audic, Y., Omilli, F., and Osborne, H.B. 1998. Embryo deadenylation element-dependent deadenylation is enhanced by a cis element containing AUU repeats. Mol. Cell. Biol. 18: 6879-6884.

Bonnet-Corven, S., Audic, Y., Omilli, F., and Osborne, H.B. 2002. An analysis of the sequence requirements of EDEN-BP for specific RNA binding. Nucleic Acids Res. 30: 4667-4674.

Detivaud, L., Pascreau, G., Karaiskou, A., Osborne, H.B., and Kubiak, J.Z. 2003. Regulation of EDEN-dependent deadenylation of Aurora A/Eg2-derived mRNA via phosphorylation and dephosphorylation in Xenopus laevis egg extracts. J. Cell Sci. 116: 2697-2705.

Dignam, J.D., Lebovitz, R.M., and Roeder, R.G. 1983. Accurate transcription initiation by RNA polymerase II in a soluble extract from isolated mammalian nuclei. Nucleic Acids Res. 11: 1475-1489.

Faustino, N.A. and Cooper, T.A. 2005. Identification of putative new splicing targets for ETR-3 using sequences identified by systematic evolution of ligands by exponential enrichment. Mol. Cell. Biol. 25: 879-887.

Ford, L.P. and Wilusz, J. 1999. An in vitro system using HeLa cytoplasmic extracts that reproduces regulated mRNA stability. Methods 17: 21-27.

Ford, L.P., Watson, J., Keene, J.D., and Wilusz, J. 1999. ELAV proteins stabilize deadenylated intermediates in a novel in vitro mRNA deadenylation/degradation system. Genes \& Dev. 13: 188-201.

Gao, M., Fritz, D.T., Ford, L.P., and Wilusz, J. 2000. Interaction between a poly(A)-specific ribonuclease and the $5^{\prime}$ cap influences mRNA deadenylation rates in vitro. Mol. Cell 5: 479-488.

Gherzi, R., Lee, K.Y., Briata, P., Wegmuller, D., Moroni, C., Karin, M., and Chen, C.Y. 2004. A KH domain RNA binding protein, KSRP, promotes ARE-directed mRNA turnover by recruiting the degradation machinery. Mol. Cell 14: 571-583.

Ho, T.H., Bundman, D., Armstrong, D.L., and Cooper, T.A. 2005. Transgenic mice expressing CUG-BP1 reproduce splicing misregulation observed in myotonic dystrophy. Hum. Mol. Genet. 14: 1539-1547.

Kahvejian, A., Svitkin, Y.V., Sukarieh, R., M'Boutchou, M.N., and Sonenberg, N. 2005. Mammalian poly(A)-binding protein is a eukaryotic translation initiation factor, which acts via multiple mechanisms. Genes \& Dev. 19: 104-113.

Korner, C.G., Wormington, M., Muckenthaler, M., Schneider, S., Dehlin, E., and Wahle, E. 1998. The deadenylating nuclease (DAN) is involved in poly $(\mathrm{A})$ tail removal during the meiotic maturation of Xenopus oocytes. EMBO J. 17: 5427-5437.

Lai, W.S., Kennington, E.A., and Blackshear, P.J. 2003. Tristetraprolin and its family members can promote the cell-free deadenylation of AU-rich element-containing mRNAs by poly(A) ribonuclease. Mol. Cell. Biol. 23: 3798-3812.
Legagneux, V., Omilli, F., and Osborne, H.B. 1995. Substrate-specific regulation of RNA deadenylation in Xenopus embryo and activated egg extracts. RNA 1: 1001-1008.

Lu, X., Timchenko, N.A., and Timchenko, L.T. 1999. Cardiac elavtype RNA-binding protein (ETR-3) binds to RNA CUG repeats expanded in myotonic dystrophy. Hum. Mol. Genet. 8: 53-60.

Lykke-Andersen, J. and Wagner, E. 2005. Recruitment and activation of mRNA decay enzymes by two ARE-mediated decay activation domains in the proteins TTP and BRF-1. Genes \& Dev. 19: 351-361.

Mukherjee, D., Gao, M., O’Connor, J.P., Raijmakers, R., Pruijn, G., Lutz, C.S., and Wilusz, J. 2002. The mammalian exosome mediates the efficient degradation of mRNAs that contain AU-rich elements. EMBO J. 21: 165-174.

Mukhopadhyay, D., Houchen, C.W., Kennedy, S., Dieckgraefe, B.K., and Anant, S. 2003. Coupled mRNA stabilization and translational silencing of cyclooxygenase-2 by a novel RNA binding protein, CUGBP2. Mol. Cell 11: 113-126.

Paillard, L., Legagneux, V., and Osborne, H.B. 1996. Poly(A) metabolism in Xenopus laevis embryos: Substrate-specific and default poly(A) nuclease activities are mediated by two distinct complexes. Biochimie 78: 399-407.

Paillard, L., Omilli, F., Legagneux, V., Bassez, T., Maniey, D., and Osborne, H.B. 1998. EDEN and EDEN-BP, a cis element and an associated factor that mediate sequence-specific mRNA deadenylation in Xenopus embryos. EMBO J. 17: 278-287.

Paillard, L., Legagneux, V., Maniey, D., and Osborne, H.B. 2002. c-Jun ARE targets mRNA deadenylation by an EDEN-BP (embryo deadenylation element-binding protein)-dependent pathway. J. Biol. Chem. 277: 3232-3235.

Paillard, L., Legagneux, V., and Beverley, O.H. 2003. A functional deadenylation assay identifies human CUG-BP as a deadenylation factor. Biol. Cell. 95: 107-113.

Philips, A.V., Timchenko, L.T., and Cooper, T.A. 1998. Disruption of splicing regulated by a CUG-binding protein in myotonic dystrophy. Science 280: 737-741.

Richter, J.D. 1999. Cytoplasmic polyadenylation in development and beyond. Microbiol. Mol. Biol. Rev. 63: 446-456.

Roberts, R., Timchenko, N.A., Miller, J.W., Reddy, S., Caskey, C.T., Swanson, M.S., and Timchenko, L.T. 1997. Altered phosphorylation and intracellular distribution of a (CUG)n triplet repeat RNA-binding protein in patients with myotonic dystrophy and in myotonin protein kinase knockout mice. Proc. Natl. Acad. Sci. 94: 13221-13226.

Takahashi, N., Sasagawa, N., Suzuki, K., and Ishiura, S. 2000. The CUG-binding protein binds specifically to UG dinucleotide repeats in a yeast three-hybrid system. Biochem. Biophys. Res. Commun. 277: 518-523.

Timchenko, L.T., Miller, J.W., Timchenko, N.A., DeVore, D.R., Datar, K.V., Lin, L., Roberts, R., Caskey, C.T., and Swanson, M.S. 1996. Identification of a (CUG)n triplet repeat RNA-binding protein and its expression in myotonic dystrophy. Nucleic Acids Res. 24: 4407-4414.

Timchenko, N.A., Wang, G.L., and Timchenko, L.T. 2005. RNA CUGbinding protein 1 increases translation of $20-\mathrm{kDa}$ isoform of CCAAT/enhancer-binding protein $\beta$ by interacting with the $\alpha$ and $\beta$ subunits of eukaryotic initiation translation factor 2. J. Biol. Chem. 280: 20549-20557.

Voeltz, G.K. and Steitz, J.A. 1998. AUUUA sequences direct mRNA deadenylation uncoupled from decay during Xenopus early development. Mol. Cell. Biol. 18: 7537-7545.

Wilusz, J. and Shenk, T. 1988. A 64 kd nuclear protein binds to RNA segments that include the AAUAAA polyadenylation motif. Cell 52: $221-228$.

Wilusz, C.J. and Wilusz, J. 2004. Bringing the role of mRNA decay in the control of gene expression into focus. Trends Genet. 20: 491-497.

Xu, N., Chen, C.Y., and Shyu, A.B. 1997. Modulation of the fate of cytoplasmic mRNA by AU-rich elements: Key sequence features controlling mRNA deadenylation and decay. Mol. Cell. Biol. 17: 4611-4621. 

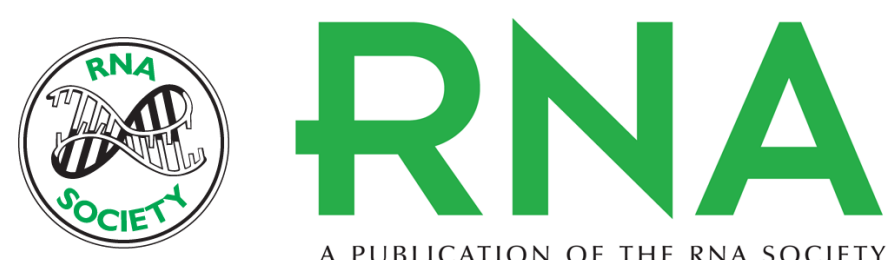

A PUBLICATION OF THE RNA SOCIETY

\section{CUG-BP binds to RNA substrates and recruits PARN deadenylase}

Karen C.M. Moraes, Carol J. Wilusz and Jeffrey Wilusz

RNA 2006 12: 1084-1091

References This article cites 32 articles, 18 of which can be accessed free at:

http://rnajournal.cshlp.org/content/12/6/1084.full.html\#ref-list-1

\section{License}

Email Alerting Receive free email alerts when new articles cite this article - sign up in the box at the Service top right corner of the article or click here.

To subscribe to $R N A$ go to:

http://rnajournal.cshlp.org/subscriptions 